\title{
Deutschunterricht für Lehramtsstudierende: die Herausforderung, durch grammatische Übungen zu motivieren'
}

\section{German lessons for student teachers: the challenge of motivating through grammatical tasks}

\author{
Monique Cunha de Araújo * \\ Fabio Anschau**
}

ZUSAMMENFASSUNG: Diese Arbeit befasst sich mit den Auswirkungen der Anwendung von offenen und halboffenen Übungen beim Unterrichten grammatischer Punkte im Fach Deutsch als Fremdsprache für Lehramtsstudierende der Fachrichtung Portugiesisch-Deutsch, vor allem in Bezug auf die Motivation im Unterricht. Mit dieser Gruppe war diesbezüglich ein gewisser Mangel an Motivation zu verzeichnen, da unter anderem bei der Festigung grammatischer Aspekte zu sehr reproduktiven Übungen eingesetzt wurden. Durch die Anwendung von offenen und halboffenen Übungen bis hin zu Grammatikfragen wurde anhand von Bewertungsbögen und Beobachtungsformular deutlich, dass diese Übungstypen auch große Hilfe bei der grammatischen Wahrnehmung der Fremdsprache sein können.

SCHLÜSSELWÖRTER: Übungenerstellung; DaF; Motivation; grammatische Wahrnehmung.

ABSTRACT: This work aims to present the results of a research on the impact of the application of open and semi-open tasks when teaching grammatical points in German as a foreign language to student teachers of Portuguese - German, especially with regard to motivation in the classroom. This group showed a certain lack of motivation in this respect, since, among other things, very reproductive exercises were used to consolidate grammatical aspects. By using open and semiopen exercises up to questions about grammar, it could be verified through assessment sheets and observation forms that these types of tasks can also be a great help in the grammatical awareness of the foreign language.

KEYWORDS: Tasks production; German as a foreign language; motivation; grammatical awareness.

\footnotetext{
* Doutoranda na linha de pesquisa Teoria, Crítica e Comparatismo do Programa de PósGraduação em Letras da Universidade Federal do Rio Grande do Sul. Bolsista do DAAD (Serviço Alemão de Intercâmbio Acadêmico), mcunhadearaujo@gmail.com, http://orcid.org/ooooooo3-0759-3614.

** Mestre em Linguística Aplicada pelo Programa de Pós-Graduação em Letras da Universidade Federal do Rio Grande do Sul. Diretor do Departamento de Ensino do Goethe-Institut Rio de Janeiro, fabio.anschau@goethe.de, http://orcid.org/oooo-0001-7644-5282.
} 


\begin{abstract}
Die von der Selbstbestimmungstheorie beschriebenen psychologischen Grundbedürfnisse nach Kompetenz, sozialer Eingebundenheit und vor allem nach Autonomie nehmen zu. Und dieser Prozess kann durch positiv wirkende Faktoren gefördert werden. Dazu zählt die Transparenz der Ziele und Wege des Unterrichts sowie der anvisierten Lernfortschritte. Zugleich sollten die Studierenden das Gefühl bekommen, in einem lernförderlichen Klima zu agieren, in dem ein gerechter und respektvoller Umgang mit dem Lehrenden und untereinander praktiziert wird. Entscheidend ist demnach eine Balance zwischen einem vertieften Verständnis für das unterrichtliche Arrangement und dem eigenen Erleben im Klassenzimmer.
\end{abstract}

(SCHART, 2019)

\section{Einleitung}

In einem Vortrag an der Cambridge-Universität im Jahr 2018 erwähnte der renommierte Professor und Sprachforscher Zoltán Dörnyei wesentliche Bedingungen für eine dauerhafte Motivation der Lernenden während des Lernprozesses einer Fremdsprache. In diesem Vortrag, Engaging language learners, zeigte Dörnyei unter anderem wie man die Motivation der Lernenden im Unterricht fördern kann. Nicht nur verteidigt er die Motivation als Strategie, um die Lernenden im Unterricht zu begeistern, sondern bekräftigt er, dass dieses Ziel erweitert werden muss und dass das Engagement der Lernenden angestrebt werden sollte. Laut dem Linguisten ist Motivation wichtig für den ersten Schritt in der Fremdsprache, aber Engagement ist sowie ein Pakt, der das Engagement für das Weiterlernen und die Autonomie, sowohl für den Lernenden als auch für den Lehrenden, festlegt: „Motivation ist notwendig, um ,den Deal vorzubereiten“, aber Engagement ist unabdingbar, um ,den Deal zu besiegeln“". (DÖRNYEI, 2018, eigene Übersetzung²).

\footnotetext{
${ }^{1}$ Dieses Projekt wurde im Rahmen der Fortbildung Deutsch Lehren Lernen vom GoetheInstitut und von der Bundesstaatlichen Universität von Bahia (Universidade Federal da Bahia) in Partnerschaft mit der Friedrich-Schiller-Universität Jena durchgeführt. ${ }^{2}$ „Motivation is necessary for ,preparing the deal', but engagement is indispensable for ,sealing the deal“".
} 
Die Aufgabe, einen Lernenden zu engagieren, hängt natürlich nicht nur mit der Arbeit des Fremdsprachenlehrers zusammen, sondern auch mit der Rolle der Institutionen und ihre Bereitschaft, einige Strukturen und Mentalitäten zu ändern. In seiner Konzeption zur Förderung engagierter Schüler bemerkt Dörnyei, dass unter anderem das Engagement mit Aufgaben (Learning Tasks) äußerst wichtig ist, da er der Ansicht ist, dass die Reflexion der behandelten Themen und die unterschiedlichen Arten der Übungen Herausforderungen anbiete. Nach diesen Vorstellungen wurde dieses Projekt idealisiert, um über einige im angewendeten Lehrwerk vorgeschlagene Aufgaben nachzudenken.

Die ursprüngliche Idee dieses Projekts war es, Lehramtsstudierende in die Lage zu versetzen, über die Themen in Deutsch als Fremdsprachenunterricht nachzudenken und sie für eine Zukunft als Lehrer zu motivieren und zu engagieren. Im Unterricht wurde festgestellt, dass einige grammatische Themen in einem Muster vorlagen, das die Lernenden nur auswendig lernen konnten. Dadurch verloren sie die Motivation und behaupteten, die Sprache sei sehr kompliziert. Dieses Projekt war nur der erste Schritt für die Studierenden, sich zu engagieren und über Themen nachzudenken, die anscheinend sehr strukturell sein würden. Dazu versuchen wir zunächst, geschlossene Übungen in offene oder halboffene zu verwandeln, da die Lernenden in diesem Aufgabenmodell freier über die strukturellen Formen der Sätze nachdenken können.

Bei der Arbeit mit Lehramtsstudierenden in Deutsch als Fremdsprache wurde beobachtet, dass es an Motivation und Interesse an der Grammatik mangelte, insbesondere bei denjenigen Aspekten, die Zeit und Mühe erfordern oder die sich von den Strukturen der Muttersprache, Portugiesisch, deutlich unterscheiden. Aus diesem Grund wurden für dieses Projekt Grammatikfragen ausgewählt, die für die Lernenden große Schwierigkeiten bereiteten, beispielsweise die Deklination von Adjektiven.

Normalerweise werden nicht nur die Deklination von Adjektiven, sondern auch viele andere grammatischen Strukturen der deutschen Sprache von den Lernenden mit der traditionellen Lernmethode erfasst: auswendig lernen. Das Ziel dieses Projekts ist natürlich nicht, diese Methode zu verwerfen, sondern die 
Verwendung unterschiedlicher Methoden im Unterricht $\mathrm{zu}$ fördern, um verschiedene Profile der Lernenden zu umfassen (vgl. GROTIAN; BEELICH, 1999, S. 50). Insbesondere zielt das Projekt darauf ab, das Bewusstsein und das Nachdenken grammatischer Strukturen in der deutschen Sprache anzuregen, um die Motivation für das Lernen dieses Themas zu erhöhen.

Damit der Einstieg neuer grammatischer Inhalte für diese Lernenden effektiv, motivierend und interessant wird, schlägt man in dieser Arbeit eine Einführung der Deklination von Adjektiven aus einer halboffenen Übung vor, in der die Lernenden dazu angehalten werden, über grammatische Strukturen nachzudenken und die sogar herauszufordern. Ebenso kann man die beiden Arten von Aktivitäten mit dieser Gruppe sowohl als eine halboffene Übung wie auch als eine geschlossene Übung durchführen, und sie mit demselben grammatischen Inhalt vergleichen.

Dieses Projekt war ziemlich aufschlussreich bei der Verwendung verschiedener Übungen im Unterricht. Darüber hinaus diente das Projekt auch zu der Stärkung der Beziehungen zwischen der Dozentin und den StudentenInnen, da die Fragestellung der Übung zuerst einen Austausch zwischen den Lernenden benötigte und schließlich die Interaktion mit der Lehrenden förderte. Das Lernklima im Unterricht wurde durch die Aktivität lockerer und die Lernenden wurden mehr und mehr auf die verschiedenen Facetten der deutschen Sprache aufmerksam. Diese Forschung wurde an der Bundesstaatlichen Universität von Pelotas (Universidade Federal de Pelotas UFPel) mit Lehramtsstudierenden des zweiten Semesters der Jahrgänge 2017 und 2018 durchgeführt.

$\mathrm{Zu}$ Beginn der Arbeit mit der Gruppe wurde bemerkt, dass die Studierenden an eine traditionelle Methode des Lernens gewöhnt waren, die ausschließlich auf einem systematischen Verstehen der Grammatik mit Tabellen, Wiederholungen und Ausfüllen von Lücken beruhte. Obwohl die vorgeschlagene halboffene Übung auf einer geschlossenen Übung des verwendeten Lehrbuchs basierte, das sehr strukturelle Merkmale aufwies, bestand die Herausforderung dieses Projekts genau darin, strukturelle Aufgaben reflektierender zu gestalten 
und zum Nachdenken anzuregen. Hierbei konzentriert dieses Projekt sich auch auf die Unterschiede zwischen geschlossenen und offenen Aufgaben und deren Auswirkungen auf die Motivation und das Interesse der Lernenden, um das Engagement der Lernenden zu erreichen. Da Lehramtsstudierende ein tieferes sprachliches und grammatisches Bewusstsein benötigen, wurde hier daran gedacht, grammatische und reproduktive Aufgaben offener zu gestalten, d. h. mit dem Ziel relevante grammatische Äußerungen zu thematisieren. Aufgrund eines ständigen Wiederholens einer grammatischen Struktur, kann das Erlernen manchmal langweilig und demotivierend sein. Die Ergebnisse der Forschung waren produktiv und stellten die Effektivität der Motivation und des Interesses der Lernenden dar.

\section{Die Übungen im Deutschunterricht in der Deutschausbildung: einige Überlegungen}

Sowohl in der Fachdidaktik als auch in der Praxis des Deutschlehrers weiß man um die Bedeutung der Übungenerstellung, insbesondere wenn man eine gute Arbeitsatmosphäre im Klassenraum anstrebt. Im Hinblick auf die Förderung von Kreativität und die Heranerziehung kritischerer Lernenden sind zweifellos die offenen Aufgabenstellungen der große Gewinner. Die Eröffnung einer Aufgabe

\footnotetext{
kann einen großen Gewinn für die Arbeitsatmosphäre in einem Klassenraum darstellen, wenn die Lernerinnen und Lerner zur Kreativität herausgefordert werden und sie sich mit paradoxer, problematischer, unerwarteter oder kontroverser Fragestellung auseinandersetzen dürfen. (SCHART; LEGUTKE, 2012, S. 105)
}

Die Erstellung einer offenen Übung kann jedoch für die Lehrenden eine Herausforderung darstellen. In diesem Sinne ist es zunächst notwendig, die Übungentypen und ihre Vorteile zu konzipieren. Bei der Anwendung dieser Aufgaben muss natürlich auch der Kontext berücksichtigt werden (SCHART; 
LEGUTKE, 2012, S. 107). Daher wurde der Versuch unternommen, die grammatischen Übungen, die normalerweise mit geschlossenen Übungen verwendet werden, wie z. B. die Deklination von Adjektiven, zu öffnen. In unserem Fall zielte dieses Experiment darauf ab, das Interesse und die Motivation durch grammatische Übungen zu wecken, denn es wurde bemerkt, dass den Lernenden die grammatischen Themen gefallen, aber nicht die Methode, wie sie lernten. Das Lernen solcher Themen war sehr mühsam und hart, weil sie vor dem Projekt dachten, sie müssten immer die ganzen grammatischen Regeln auswendig lernen. Das Ziel war es also, die Idee, dass die Grammatikregeln auswendig gelernt werden sollen, zu entmystifizieren.

Bei diesem Versuch bot die Anwendung der offenen Übungen eine gute Möglichkeit, weil sie die subjektive Bewertung einer bestimmten Idee durch die Lernenden zu begünstigen sind (SCHART; LEGUTKE, 2012, S. 106). Darin werden die Lernenden herausgefordert, über ein bestimmtes Thema zu reflektieren, was ihre Kritik- und Analysefähigkeit fördert. Natürlich trägt nicht nur die einfache Idealisierung einer offenen Übung zum Aufbau eines Gedankenaustausches oder zur Argumentation bei, jedoch eine verstärkt handlungsorientierte Aufgabe begünstigt effektiv die Kommunikation (vgl. BOHL; KUCHARZ, 2010; KLEINKNECHT, 2010).

In den didaktisch-methodischen Prinzipien stellt man hinsichtlich der Erstellung einer Übung verschiedene Typen fest. Die handlungsorientierten Übungen sind beispielsweise auf die Idee des Handelns mit der Sprache gerichtet:

Das heißt nicht nur, andere verstehen und sich verständlich machen, sondern auch Bedeutungen von Wörtern oder Gesten aushandeln, argumentieren, Inhalte zusammenfassen, Stellung nehmen, Meinungen ausdrücken oder auf Meinungen eingehen. (FUNK et al., 2012, S. 18)

An diesem Punkt wird wahrgenommen, dass das handlungsorientierte Prinzip nicht nur an der kommunikativen Praxis im Allgemeinen ausgerichtet ist, sondern auch die Optimierung der kritischen Idealisierung der Lernenden anstrebt. In Bezug auf die reflexive Konstruktion von Fremdsprachenstudenten helfen handlungsorientierte Übungen bei der Suche nach Argumenten für eine 
bestimmte Idee, die verteidigt oder widerlegt werden soll. Nach unserem Verständnis jedoch können handlungsorientierte Übungen sich in einem kulturellen Bereich befinden - wenn sich die Übungen auf einen spezifisch kulturellen Kontext beziehen, wie im Fall von Landeskunde-Übungen - oder auch in einer der Lebenswelt gelegene Sphäre, wie folgt:

\footnotetext{
Den Lernenden sollten in einem handlungsorientierten Unterricht Situationen und Themen angeboten werden, die für ihre Lebenswelt bedeutungsvoll sind. Die Situationen, in denen die Lernenden im Unterricht handeln, sollten so auch außerhalb des Unterrichts vorkommen können. (FUNK et al., 2012, S. 18)
}

Diese Art von Unterricht bzw. Übungen müssen die Lernenden in ihrer Lebenswelt lokalisieren, sodass diese Art von Übung für sie sinnvoll ist. In unserem Fall wäre die Lebenswelt der Lehramtsstudierenden ebenfalls mit einem außerhalb des Unterrichts verbunden, aber auch Situationen und Themen sollten auf ihre zukünftige Praxis als Deutschlehrende abgestimmt sein. Das bedeutet mit anderen Worten, dass den Studierenden der Deutschausbildung handlungsorientierte Übungen für ihre Praxis angeboten werden sollten, da diese einem Ideal von bedeutungsvoll für ihre Lebenswelt entsprechen.

Aus diesen Ideen lässt sich ableiten, dass die Übungen, wie erwähnt, neben der Positionierung der Lernenden in einer zukünftigen Lebenswelt auch in Bezug auf ihre Funktion - reflektieren als auch argumentieren - die Grundlagen für die Bildung und Konstitution dieser zukünftigen Lebenswelt bilden. Aus dieser Auffassung geht natürlich hervor, dass die Übungen auch eine grammatische Vertiefung enthalten müssen, d. h. sie müssen sich auch auf die konstitutiven Formen der grammatischen und sprachlichen Basis beziehen. Offensichtlich geht es nicht darum, nur und ausschließlich eine einzige theoretische Grundlage für die Erstellung der Übungen für dieses Publikum zu verteidigen, sondern ist es hier zu versuchen, die traditionelle Methode der Verzierung der angewandten Grammatikregeln anders zu verstehen und anzubieten. In diesem Sinne wird auch angenommen, dass abwechslungsreiche Übungen für diese Lernenden ideal sind, da sie mit verschiedenen Übungsformen in Kontakt treten können und somit eine größere Anzahl von Lernendenprofilen umfassen. 
Wenn man sich auf die Übungen bezieht, die sich an die Lernenden einer Deutschausbildung richten, müssen wir, wie bereits erwähnt, berücksichtigen, dass sich die Handlungsorientierung auch auf das Arbeitsumfeld bezieht. Auch diese Umgebung muss in unserem Fall so strukturiert sein, dass die Form der Grammatik gleichfalls fokussiert werden kann. In diesem Sinne glauben wir, dass bei einer intensiven Anwendung von Übungen auf die reine grammatische Aufgabe gesagt werden kann, dass die formfokussierte Übungen Teil einer ersten Phase sind, um dieses Ziel zu erreichen, denn

eine sinnvolle Abfolge von inhaltsbezogenen $\mathrm{zu}$ formorientierten Übungen ist unserer Meinung nach schon allein durch die menschliche Neugier vorgegeben: Wir alle lesen oder hören Texte, um die Inhalte zu verstehen, nicht um neue sprachliche Formen zu erarbeiten. Erst wenn die Inhalte eines Textes den Lernenden klar sind und sie wissen, worum es im Text geht, können sie sich auf neue Formen konzentrieren. (FUNK et al., 2012, S. 21)

Obwohl die DaF-Studierenden in Brasilien oft mit einem anfänglichen Sprachniveau der Fremdsprache das Studium beginnen, ist es klar, dass die Erfassung von Inhalten in gewisser Weise erleichtert wird, wenn man ihnen Leseübungen anbietet. Auf der anderen Seite versteht man, dass diese Studierenden gleichzeitig den Inhalt eines Textes verstehen sollten, d. h. sich auch auf seine Struktur konzentrieren sollten. Wenn man einfache Lückenübungen oder andere geschlossene Übungen hat, die normalerweise nach einer Kontextualisierungsaktivität eingefügt werden, werden die Lernenden mit der Form und ihren Regeln konfrontiert, da die geschlossenen Übungen einen reproduktiven Charakter haben (FUNK et al., 2012, S. 31).

In diesem Sinne ist ein anderes methodologisches Prinzip wie die Personalisierung diesem Profil angemessen. Im Fall der Erstellung von Material für Studierende der Deutschausbildung ist auch das Bewusstsein für diese Strukturen äußerst relevant. Mit diesem Ziel wurde diese Forschung idealisiert, ausgehend von der Annahme, dass die formfokussierten Übungen in gewisser Weise auch handlungsorientiert sein können und durch offene Übungen die 
Reflexion fördern können, wobei der Schwerpunkt auf der Motivation im Unterricht und der Interessenförderung liegt.

\title{
3 Motivation als Motor des Fremdsprachenunterrichts
}

Die Motivation im Unterricht ist ein Thema, das in der Fachdidaktik für DaF im Laufe der Jahre bereits untersucht und diskutiert wurde. Roths Aufsatz befasst sich mit der gemeinsamen Arbeit von Lehrenden und Lernenden. Das Bestreben, die Motivation $\mathrm{zu}$ fördern, beruht laut Neurowissenschaftler hauptsächlich auf der Arbeit des Lehrers. Motivierte Lernenden sind in der Lage, Strukturen länger im Gehirn gespeichert zu halten, insbesondere wenn sie emotional betroffen sind:

\begin{abstract}
Interesse und Motiviertheit 3 drücken sich in Erhöhung des noradrenergen Systems, das die allgemeine Aufmerksamkeit erhöht (leichter Erwartungsstress), des dopaminergen Systems (Neugier, Belohnungserwartung) und des cholinergen Systems (gezielte Aufmerksamkeit, Konzentration) aus. Diese Systeme machen die Großhirnrinde und den Hippocampus bereit zum Lernen und fördern die Verankerung des Wissensstoffes im Langzeitgedächtnis. Wie dies genau passiert, ist nicht bekannt. Bekannt ist hingegen, dass die Stärke des emotionalen Zustandes, den der Schüler als Interesse, Begeisterung, Gefesseltsein empfindet, mit der Gedächtnisleistung positiv korreliert. (ROTH, 2004. S. 503)
\end{abstract}

Die Begriffe Motivation und Motivierung mögen im Duden ähnlichen semantischen Wert haben, aber ihre Anwendungen unterscheiden sich in der angewandten Sprachforschung. Für diese Untersuchung ist die genauere Erfassung dieser beiden Formen wichtig. In der Fachliteratur wurde die Verwendung der beiden Formen in unterschiedlicher Weise verifiziert. Genauer

\footnotetext{
3 Interessant ist in diesem Zusammenhang der feine Unterschied zwischen Motivation und Motivierheit: „Immer häufiger wird statt Motivation eher Motiviertheit genutzt. Beides steht im Duden, beides bedeutet im Prinzip das Gleiche. Motiviertheit soll jedoch besonders die Dauerhaftigkeit und Dynamik des motivierten Zustands beschreiben." (FRANK, Moira. Motivierung vs. Motivation. Hamburg: Dashöfer, 2018. Verfügbar unter: https://www.dashoefer.de/newsletter/artikel/motivierung-vs-motivation.html Zuletzt abgerufen: 24 Mai 2019).
} 
gesagt hat die Motivation mit dem Zustand selbst zu tun, während die Motivierung mit dem eigentlichen Motivationsprozess einhergeht (STARK; MANDL, 2000, S. 96). Wenn in dieser Studie die Erhöhung der Motivation erwähnt wird, wird auf den allgemeinen Zustand der Lernenden verwiesen, die nicht motiviert waren. Was den Prozess der Motivation betrifft, bezieht er sich auf den Zweck der Motivierung. Das Konzept der Motivation wird in zwei weitere Arten unterteilt: die Extrinsische und die Intrinsische Motivation. Die beiden Begriffe sind für eine Forschung der Motivationstheorie und der Selbstbestimmungstheorie am relevantesten. (vgl. RIEMER, 2010; KOUSALOVÁ, 2018).

Die extrinsische Motivation bezieht sich direkt auf das Ende der Motivation, nämlich auf das, was die Lernenden als Belohnung erhalten, z. B. die guten Noten. In diesem Sinne werden die Lernenden von einem Abschlusspreis motiviert, der so etwas wie einen motorischen Mechanismus für die Motivation darstellt. Diese Auszeichnungen können von jeglicher Art sein, sind äußerlich und entstehen nicht aus einer intimen Manifestation des Willens. Andererseits hat die intrinsische Motivation mit Aktivität zu tun, die vom Willen getrieben wird, der von Neugier befriedigt wird. Diese Art der Motivation steht in direktem Zusammenhang mit dem Interesse der Lernenden. Wie Riemer ausführt, liegt der Unterschied zwischen beiden Motivationsarten vor allem in der Potenz des inneren Interesses und im lohnenden Interesse:

Extrinsisch motivierte Lernende benötigen Anreize, die außerhalb der Lernaufgabe liegen (z. B. Belohnungen aller Art, auch: gute Noten), während intrinsisch Motivierte aus innerem Bedürfnis, Interesse und Vergnügen eine Zielsprache Lernen. (RIEMER, 2010, S. 170)

Deshalb wird die intrinsische Assoziation von Interesse und Motivation als Motor für innere Zufriedenheit im Hinblick auf die Motiviertheitssensation verstanden. Aus diesem Grunde berücksichtigte dieses Projekt nicht nur die Frage, ob die Motivation durch halboffene Übungen erhöht werden soll, sondern auch, ob diese Übungen das Interesse der Lernenden an dem behandelten Thema auf irgendeine Weise geweckt haben. Es wurde verstanden, dass es nicht möglich 
sein würde, die Begriffe Interesse von Motivation zu trennen, da die beiden Konzepte, wie hier schon erwähnt, in einer engen Beziehung zueinanderstehen.

\section{Interesse und Motivation durch (halb)offene Übungen?}

Die Motivation und das Interesse, die vom Lehrer ausgehen, sind in diesem Zusammenhang wichtig, da sie das Lernen anregen. Natürlich ist nicht nur die Motivation des Lehrers für die Aufrechterhaltung eines guten Lernklimas verantwortlich, sondern es gibt eine Reihe von Techniken und Methoden, die den Unterricht interessanter machen können. In der Tat glauben manche Lehrer, dass Motivationskurse nur durch Spiel und Verspieltheit erreicht werden können; andererseits weiß man, dass sie auch durch herausfordernde und reflektierende Übungen erreicht werden können (SCHART; LEGUTKE, 2012, S. 10). Diese Übungen sollten die Lernenden jedoch emotional erreichen (vgl. ROTH, 2003).

Die Idee einer Übung, die die Lernenden auf persönliche Weise erreicht, kann als eine Vorstellung von Personalisierung bezeichnet werden. Die Personalisierung ist einer der wichtigsten Faktoren, um die Motivation und das Interesse an einer Übung zu steigern, wie Hermann Funk et al. nahelegen:

\footnotetext{
Bei der Personalisierung [...] geht es darum, die Motivation beim Lernen zu erhöhen und das Gelernte besser zu behalten. Dazu sollten Übungen und Aufgaben einen persönlichen Bezug zu den Lernenden haben, und die Lernenden sollten aktiv in den Unterricht mit einbezogen werden. (FUNK; KUHN et al, 2012, S. 22)
}

In einer kreisförmigen Vorstellung des Motivationsprozesses im DaF Unterricht, die Dörnyei im Abbildung 1 vorschlägt, wird bezeichnet, dass das vom Lehrer hergestellte Material extrem relevant ist, insbesondere wenn es mit seiner Personalisierung zusammenhängt. Nach Funk und Kuhn et al. berücksichtigt dieser Prozess auch die Relevanz dieses Materials für die Lernenden, in erster Linie bei der Schaffung realistischer Überzeugungen im Lernprozess. 
Abbildung 1: Komponenten des motivationsfördernden Lehrens im Fremdsprachenunterricht

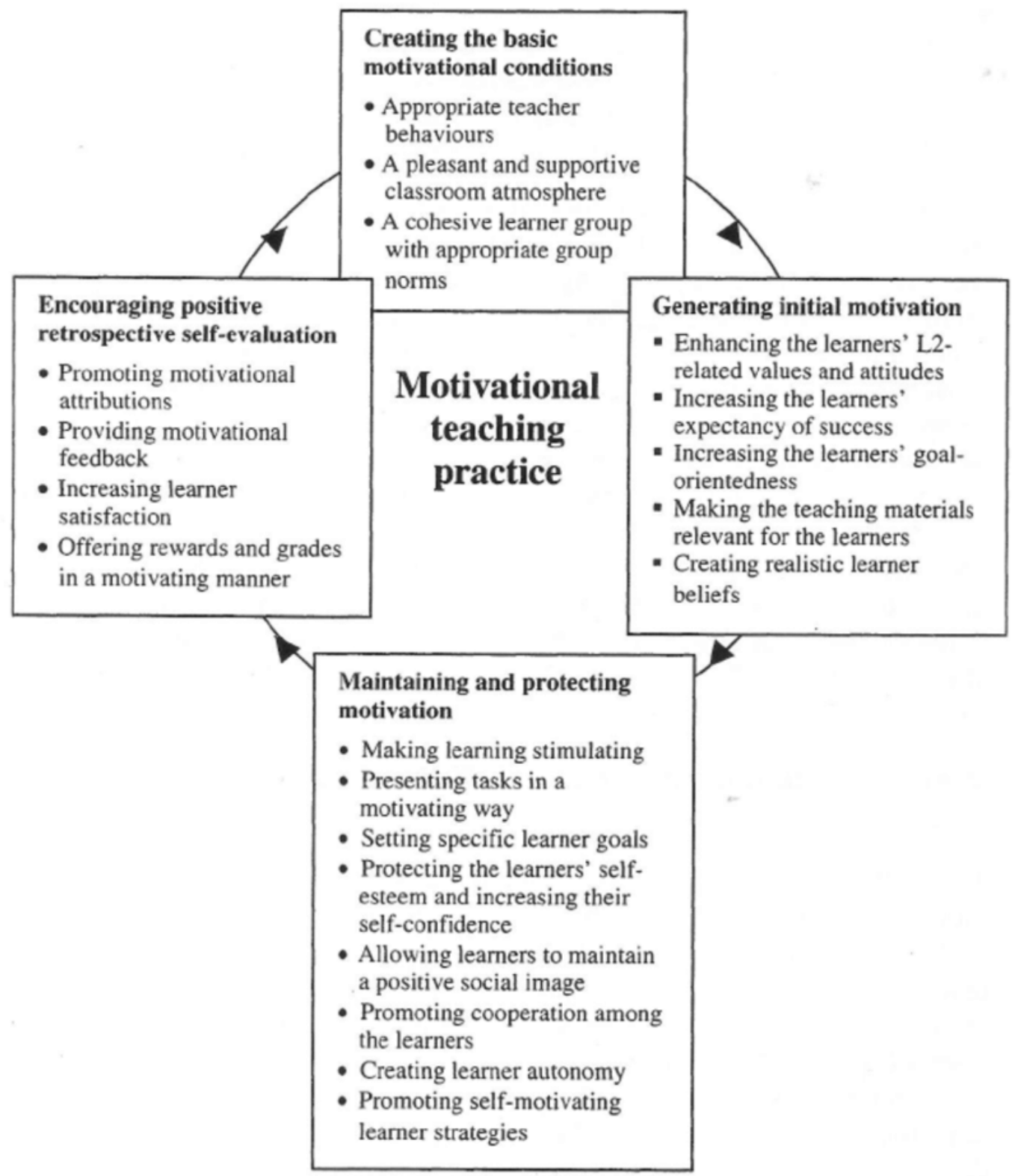

Quelle: DÖRNYEI apud KOUSALOVÁ, 2018, S. 38

Nach Dörnyeis Festlegungen könnte man in diesem Zusammenhang sagen, dass man in einer persönlicheren Übung Beispiele für Übungen mit individualisierten Fragen hat, wie beispielsweise: Was haben Sie heute gemacht? oder was würden Sie tun, wenn Sie im Lotto gewinnen würden? Diese Fragen gehen von einer Idee offener Übungen aus, auf die man zuvor hingewiesen hat. 
Aus dieser Perspektive wird angenommen, dass die Idee einer offenen Aufgabe eine einfache Lösung für die Reflexion grammatikalischer Fragen im Fall von Lehramtsstudenten sein kann, denn "offene Aufgaben geben den Lernenden viel Freiheit. Sie können ihre ganze Kreativität einsetzen und sind nicht beschränkt auf bestimmte Denkwege oder Interpretationen“ (INSTITUTO GOETHE, [200?]).

Bei offenen Fragen kann ihre Verwendung in Übungen viele Vorteile anbieten, insbesondere bei der Lösung von Problemen, mit denen die Lernenden konfrontiert sind, wie Wienert sagt: „Vermehrt offene Fragen einzusetzen ist auch eine Form, kompetenzorientiert zu unterrichten und Schüler/innen dazu anzuhalten, Problemlösungen in variablen Situationen erfolgreich [zu] nutzen (2003, S. 27)“. Im Falle von Lehramtsstudierenden können diese Fragen die Studierenden in der Regel auf die Karriere vorbereiten, da diese Lernenden in der Zukunft auch problematische Fragen von ihren Lernenden erhalten. Solche

\footnotetext{
Fragestellung schafft nicht nur Denkmöglichkeiten als (geschlossene Übungen), sondern öffnet auch den Raum für einen intensiven Austausch über unterschiedliche Interpretationen und Ideen. Das kann zur Entwicklung eines lernförderlichen Klimas beitragen. (SCHART; LEGUTKE, 2012, S. 107)
}

Mit anderen Worten kann man sagen, dass solche Übungen, bei denen offene Fragen von Studierenden gestellt werden, andere Denkmöglichkeiten eröffnen, die Personalisierung von Antworten anregen, ein besonderes Interesse (“zur Entwicklung eines lernförderlichen Klimas beitragen”) der Lernenden fördern und dadurch die Motivation im Unterricht steigern. Außerdem, wenn die Antworten eine Individualisierung aufzeigen, erhöhen sich die Chancen des Informationsaustauschs zwischen den Lernenden, und "wenn das gelingt, werden sie die Deutsche Sprache im Sinne des Prinzips der Handlungsorientierung aktiv im Unterricht gebrauchen" (SCHART; LEGUTKE, 2012, S. 107).

In diesem Sinne wurde mit diesem Projekt versucht zu zeigen, dass offene und auf die Grammatik ausgerichtete Aktivitäten in jeder Hinsicht zur Bewusstseinsbildung für die grammatische Struktur und auch zum Aufbau von 
Wissen über einen bestimmten grammatischen Punkt beitragen können. Ziel dieser Arbeit ist es auch, die Reflexion zukünftiger Lehrer zu fördern, die auf intuitive Weise auch solide Kommunikationsgrundlagen bieten können. Es ist wichtig zu betonen, dass die halboffene und offene Aufgabe große Möglichkeiten bietet, dieses Potenzial anzuregen.

\section{Methodologie}

Diese Forschung zielt darauf ab, grammatische Aufgaben reflexiver und kritischer zu gestalten, bezüglich sowohl der Bedeutung von Grammatikkonzepten, als auch des Aufbaus der Fremdsprache, um so ein grammatisches Bewusstsein bei den Studierenden zu erreichen. Dazu wurden Unterrichtsbeobachtungen und anschließende Befragungen der Lernenden durchgeführt.

\section{1 Beobachtungsbogen und Fragenbogen}

Um das Ausmaß des Interesses, der Motivation und auch des Engagements genauer beurteilen zu können, das durch mögliche Fragen von Lernenden an KollegInnen oder an Lehrkraft sowie durch Unterstützung gegenseitig überprüft werden konnte, wurden zwei Bewertungsformulare entwickelt. Das erste Formular besteht aus der Bewertung der Lernenden nach der Durchführung der Aktivität, das Zweite war in der Verantwortung der Lehrkraft, der nach der Beobachtung der Stimmung im Klassenzimmer die genannten Einträge markieren sollte. Die Ergebnisse wurden unmittelbar danach in einer Vergleichstabelle zur Verfügung gestellt.

Am Ende sollten die Studierenden ein Evaluationsformular ausfüllen, in dem sie die Übungen bewerten sollten. Es gab drei Möglichkeiten auf dem Formular (siehe Anhang): Interesse wecken, Motivation und Konzentration, und 
sie sollten „gut“, „es geht“ und „nicht gut“ ankreuzen. Dieses Formular ist auch sehr wichtig für die Reflexion der Lernenden über die Aufgaben, die zum Zeitpunkt der Markierung ausgeführt wurden. Der Lernende konnte beide Aktivitäten bewerten und reflektieren, wobei er sich an den Fragen orientierte.

Während der Aktivität wurde von der Lehrkraft auch einen Beobachtungsbogen (siehe Anhang) durchgeführt, in dem die Aufmerksamkeit, die Konzentration, die Motivation und das Interesse der Lernenden an beiden Aktivitäten überprüft werden konnten. Für den Beobachtungsbogen wurde gedacht, dass die Stimmung ausgewertet werden sollte, daher setzten wir folgende Beobachtungspunkte ein: Konzentration, Interesse, Ablenkung, Langeweile und Andere, dieses Letztere wurde in dem Bogen eingesetzt für den Fall, dass andere Situationen vorkommen würden. In diesem Sinne wurde während der gesamten Aktivität auch bewertet, ob die Lernenden Fragen stellten und Interesse an dem Thema zeigten. Die Fragen könnten nach Wiederholung oder eine Frage sein, die mit der Übung zu tun hätte. Schließlich sollten wir auf dem Formular auch markieren, wenn die Lernenden Fragen zu anderen Themen stellten. Denn es wäre für die Forschung auch wichtig zu analysieren, wie sich die Lernenden im Klassenzimmer verhalten. Deswegen würden wir durch die Beobachtung auf dem Beobachtungsbogen auch markieren, ob sie alleine beziehungsweise in Stille arbeiteten.

Das Ende der Aktivität war auch ein wichtiger Moment für die Bewertung der Lernenden. Es wurde beobachtet, ob die Lernenden noch motiviert waren, ob sie sich gegenseitig oder den Lehrer fragten und ob es eine Frage zum Thema gab, was Interesse zeigte. Natürlich basierte die Messung dieser Themen nur auf der Beobachtung dieser Punkte durch die Bewertung der Stimmung im Klassenzimmer nach der Aktivität. 


\section{Durchführung}

Die Durchführung des Projekts war nicht ganz einfach. Erstens sollte man an eine grammatische Übung denken, die irgendwie offen wäre, aber die offenen Übungen haben konzeptionell eine offene Lösung, bzw. „es gibt viele verschiedene Lösungsmöglichkeiten“ (FUNK; KUHN et al, 2012, S. 30). Daraus ergab sich eine erste Schwierigkeit bei der Vorbereitung, denn wir wussten schon aus unserem Studium, dass eine grammatische Frage immer eine richtige Lösung hat. Dann dachte man, dass eine Reflexion der Grammatik eine gute Idee wäre, wenn es um die Arbeit mit Erwachsenen geht. Wie bereits erläutert, wurde diese Arbeit mit Studierenden der Bundesstaatlichen Universität von Pelotas (UFPel) durchgeführt. Diese Studierenden befanden sich auf Niveau A1.2 (Nach dem Gemeinsamen europäischen Referenzrahmen für Sprachen - GER) und waren Menschen verschiedener Alter.

Die Lehramtsstudierenden lernen normalerweise auf eine traditionelle Weise, d. h. sie lernen die grammatischen Regeln auswendig und machen geschlossene Aufgaben, vor allem Übungen, in denen die Lücken ausgefüllt oder Sätze gebildet werden müssen. In diesem Sinne spielt die grammatische offene Aufgabe eine wichtige Rolle, denn sie ermöglicht die Reflexion der Grammatik und ihrer Regeln. Wie oben erwähnt, war die Erstellung dieser Aufgabe eine Herausforderung, deswegen fiel die Entscheidung, eine halboffene Übung zu erstellen. Auch mit diesem Übungstyp sollte es möglich sein, das Interesse für das grammatische Thema im Unterricht zu wecken sowie die möglicherweise erhöhte Motivation der Lernenden beobachten zu können.

Als Vorbereitung dachte man an zwei verschiedene Übungen für dasselbe grammatikalische Thema: eine offene Übung und eine geschlossene. Zunächst wurde die offene Aufgabe, zu einem späteren Zeitpunkt die geschlossene Aufgabe erteilt. Es wurden drei Unterrichtseinheiten zur Verfügung gestellt. In der ersten hat man durch Realien das Thema Mode und damit die Verwendung von Farben und Kleidung angesprochen. Jeder präsentierte seine Lieblingsfarbe und seine 
Kleidung, in dem Versuch, die soziale Realität im Umgang mit grammatischen Fragen zu betrachten und damit die Möglichkeit eines kommunikativen Funktionierens der Sprache zu bieten, wie wir es, wie im Lehrwerk vorgeschlagen, mit einer Deklinationstabelle tun würden. Diese Phase war daher sehr wichtig, damit die Lehrenden die bereits gelernte Strukturen auf intuitive Weise verwenden konnten, um die anfängliche Visualisierung einer Tabelle zu verhindern, die den Prozess der kommunikativen Konstruktion umkehren und behindern würde. Mit der Beschreibung der Kleidung und ihrer Farben konnten sie die Erinnerung an Farben und Kleidung aktivieren, die in früheren Semestern behandelt wurden. Dann führten sie eine Aufgabe aus, um die Farben und die Kleidung zu festigen. Bald darauf wurde die erste Aktivität des Projekts direkt ausgeteilt: eine (halb)offene Übung. In dieser Übung wurden die Lernenden zum ersten Mal mit der Struktur der Deklination der Artikel konfrontiert. In der vorangegangenen mündlichen Übung wurde viel über diese Struktur gesprochen, es wurde jedoch nicht gesagt, wie ihre Bildung funktioniert. Wie in der ersten Übung gezeigt, wurden die Lernenden eingeladen, die Ausdrücke zu lesen (vollständige Übung siehe Anhang).

Abbildung 2: (halb)offene Aufgabe - Teil 1

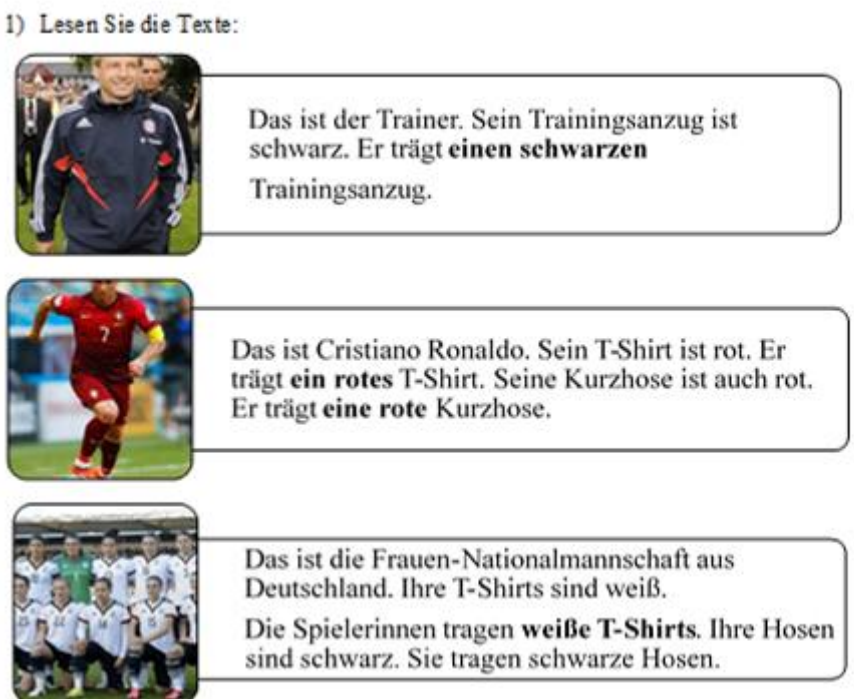

Quelle: FUNK; KUHN, 2013, S. 38 
Bald nach dieser Lektüre wurden die Teilnehmer darum gebeten, diese Strukturen zu diskutieren und ihre Bildung zu bewerten.

Abbildung 3: (halb)offene Aufgabe - Teil 2

2) Vergleichen Sie die Wörter, die fett geschrieben sind. Raten Sie mal, warum sie so aussehen?

3) Erkennen Sie diese grammatikalische Struktur? Was drückt sie aus?

4) Tauschen Sie sich Ihrer Nachbarin/Ihrem Nachbarn aus. Was sagen Sie über diese Struktur? Wie erkennt man sie im Satz? Wie wichtig ist sie?

Quelle: selbst erstellt

Mit den Fragen konnten die Lernenden erst über die grammatischen Strukturen nachdenken, bevor sie festgelegt wurden, und so eine reflektierende Umgebung zum grammatikalischen Training begünstigen. Um das Prinzip der Handlungsorientierung zu fördern, wurde mit der letzten Frage ebenfalls einen Ideenaustausch am Ende vorgeschlagen. Wie bereits erwähnt, sollte versucht werden, den Grad der Motivation und des Interesses an der Wahrnehmung zu messen und das Bewusstsein für Grammatik zu fördern, die in Lehrwerke häufig nur strukturell beschrieben werden.

Um die Vorstellung der Strukturen zu stärken, wurde eine geschlossene Übung durchgeführt, um die Motivation und das Interesse für diese Strukturen auch vergleichen zu können. Das Ideal wäre natürlich, wenn die Lehrkraft zwei verschiedene Klassen desselben Niveaus haben könnte, und dass jeweils eine Übung in einer Klasse angewandt werden könnte. Leider war dies nicht möglich, da zu Beginn nicht bekannt war, welche Fächer an jeden Lehrer verteilt wurden. Nach dem Unterricht über Kleidung und einer kurzen Einführung, die auf intuitive Weise die Deklination von Adjektiven behandelte, kamen die offenen Übungen nur als Vorentlastung, um die Lernenden dazu zu bringen, über die im 
Lehrwerk enthaltenen grammatischen Regeln nachzudenken. In jedem Fall war die Anwendung der Aktivität wichtig, um die Motivation und das Interesse der Studierenden, Hauptziel des Projekts, zu erhöhen, wie nachstehend gezeigt wird.

Es ist wichtig zu erwähnen, dass beide Übungen das gleiche grammatische Thema aus dem Lehrwerk Studio 21 A1.2 behandelten: Adjektive im Akkusativ mit unbestimmtem Artikel. Das Lehrwerk hat eine sehr interessante Struktur in Bezug auf die Darstellung der Grammatik, die schrittweise eingeführt wird, und wie im Ping-Pong-Modell kehren die Strukturen und Formen zu einem anderen Zeitpunkt im Buch auf fortgeschrittene Weise zurück. Der erste Schritt der geschlossenen Übung ist wie folgt (für die vollständige Übung, siehe Anhang):

\section{Abbildung 4: geschlossene Aufgabe - Teil 1}

1) Adjektive im Akkusativ mit unbestimmtem Artikel:

\begin{tabular}{l|lll}
\multicolumn{2}{c}{ den } & \multicolumn{1}{c}{ das } & die \\
\hline Singular & $\begin{array}{c}\text { Einen schwarzen } \\
\text { Trainingsanzug }\end{array}$ & Ein gelbes T-Shirt & Eine blaue Hose \\
Plural & Schwarze Anzüge/ T-Shirts/Hosen
\end{tabular}

Quelle: selbst erstellt mit Informationen aus FUNK; KUHN, 2013, S. 38

Mit dieser Aktivität wurden die Lernenden durch eine Deklinationstabelle in eine traditionelle grammatikalische Struktur eingeführt. Bald darauf wurden sie ermutigt, die folgenden Tabellenelemente in der folgenden Übung auszufüllen. Die Übung wurde kurz nach der Pause von 20-25 Minuten durchgeführt. Ihnen wurde gesagt, sie sollten sich nicht das vorherige Blatt ansehen, sondern sich nur auf die verteilte Tabelle konzentrieren. 
Abbildung 5: geschlossene Aufgabe - Teil 2

2) Ergänzen Sie:

einen schw arzen - eine rote - ein rotes - wed
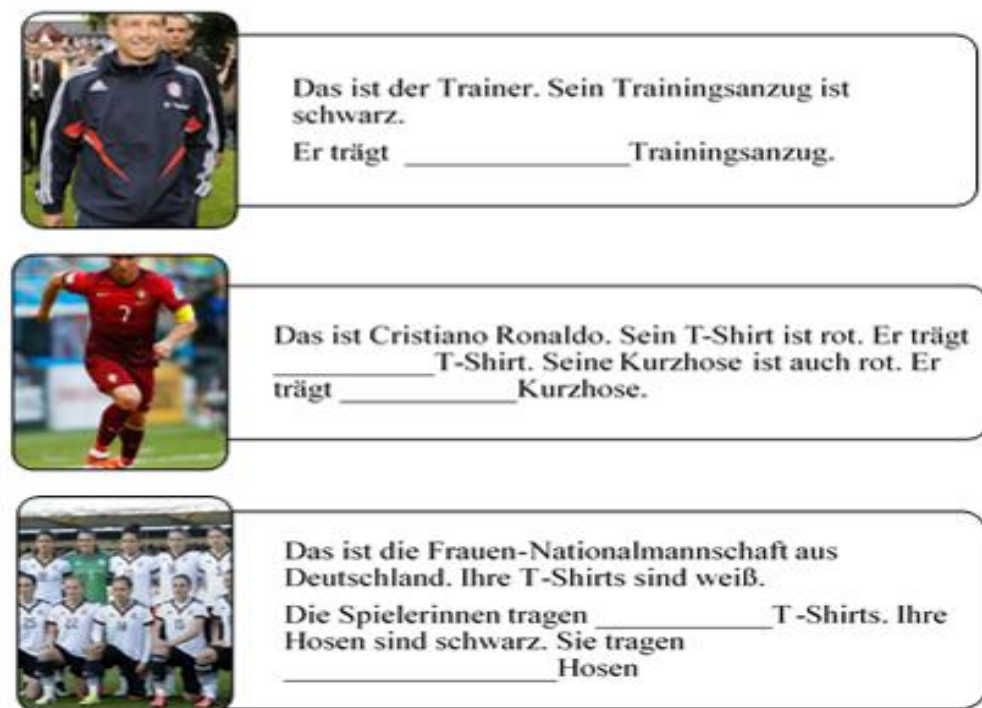

Quelle: selbst erstellt mit Informationen aus FUNK; KUHN, 2013, S. 38

Bei diesen beiden Aktivitäten war es sehr wichtig zu erkennen, dass eine geschlossene Aufgabe wirklich „keine besonders kreative Leistung erfordert. Die Lernenden müssen einem logischen, planmäßigen Denkprozess folgen. Dieser steuert, wenn er erfolgreich verläuft, direkt auf die eine Lösung $\mathrm{zu}$, die der Lückentext zulässt (SCHART; LEGUTKE, 2012, S. 106). Dies steht im Gegensatz zur (halb)offenen Aktivität, die den Austausch und die Reflexion tatsächlich begünstigte. Am Ende der beiden Aktivitäten wurden die Lernenden aufgefordert, die beiden Übungsformen zu vergleichen, und sie konnten über ihre Erfahrungen berichten. 


\section{Ergebnisse}

Die Ergebnisse waren sehr positiv. Man konnte die Wirkung der beiden Übungen vergleichen. Man konnte sehen, dass an der Übung, die aus Lücken und grammatikalischer Erklärungen bestand, die Lernenden mehr Interesse an dem grammatischen Thema zeigten und dass sie hart arbeiteten, um die Aufgabe zu lösen.

In der halboffenen Übungstellung bemerkte man, dass die Studierende viel miteinander gesprochen haben, sowohl über die Grammatikregeln und die Übungen, aber auch über andere Themen. So konnte man sehen, dass die Aufgabe nicht nur die Motivation erhöhte, sondern auch für die Interaktion der Lernenden von positivem Einfluss war. Bei der geschlossenen Aufgabe zeigten die Lernenden jedoch einen höheren Grad an Konzentration im Vergleich zu der halboffenen Aufgabe. Die Abbildung und die Daten befinden sich in der folgenden Tabelle.

Tabelle 1: Halboffene Aufgabe - Lernender Bewertung

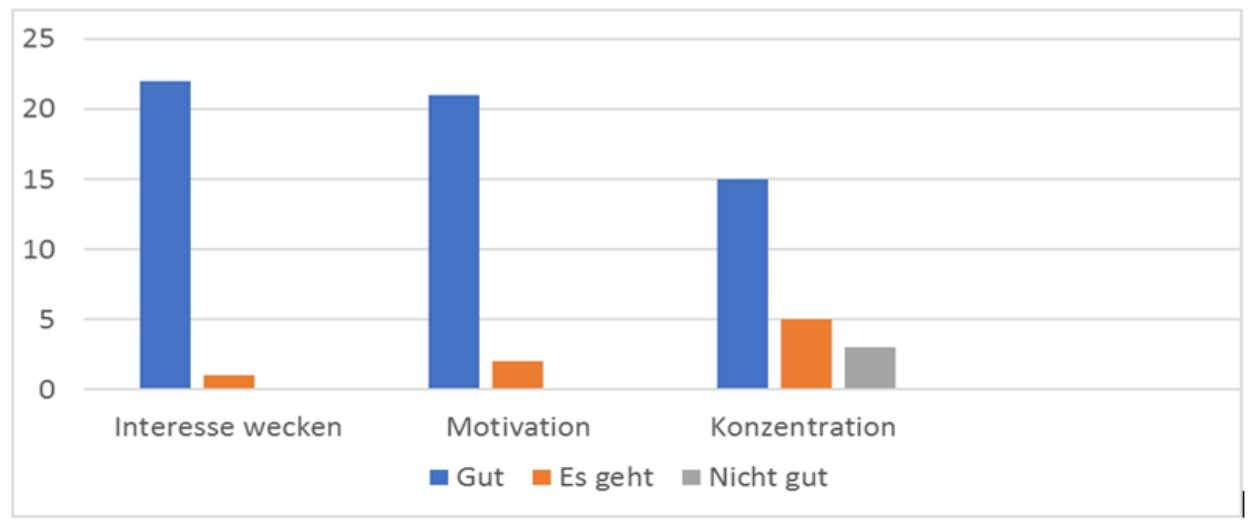

Quelle: selbst erstellt 
Tabelle 2: Geschlossene Aufgabe - Lernender Bewertung

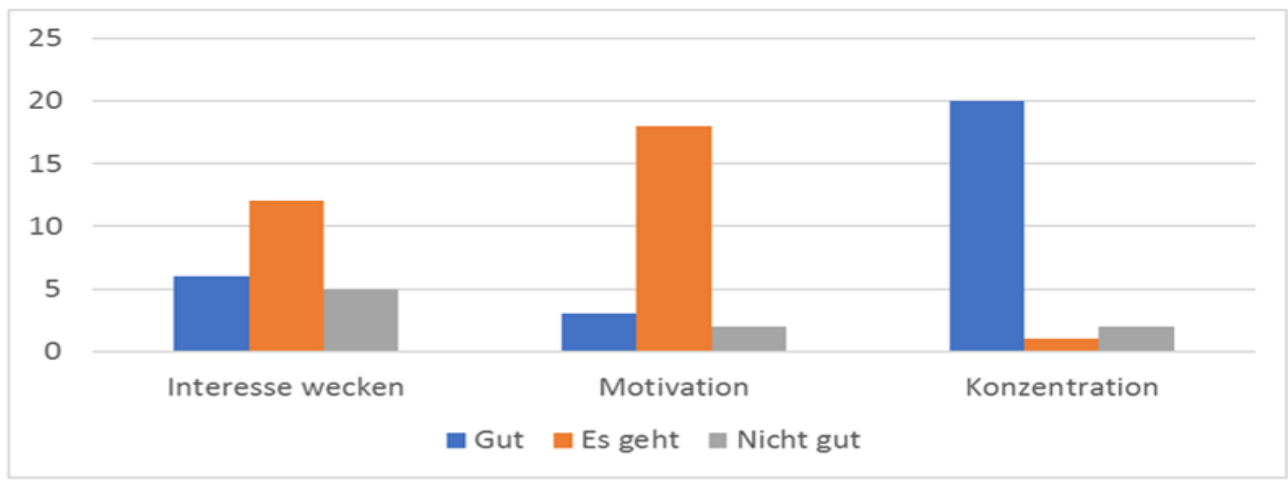

Quelle: selbst erstellt

\section{Bewertung}

Die Forschung war aus didaktischer Sicht recht interessant und hat bestätigt, dass das Interesse der Lernenden besonders durch die Abwechslung von Übungstypen geweckt wird. Es wurde auch festgestellt, dass sich die Lernenden nach der (halb)offenen Übung sicherer fühlten, um die Lücken auszufüllen und ebenfalls daran interessiert waren, nicht nur die richtige Antwort zu geben, sondern auch, die Formen und die Struktur zu vergleichen. Wir mussten jedoch berücksichtigen, dass, obwohl die (halb)offenen Übungen auch im dem Fall nur einen Blick auf die Grammatik bieten, ohne jedoch allgemein auf die kommunikative Wahrnehmung zu beachten, bestand die Idee in dieser Arbeit darin, eine Reflexion der grammatischen Strukturen zu bieten: Vorschläge für die Lehrwerke und damit prüfen, ob wir als Einführung die strukturellsten Themen wie die Deklination von Adjektiven verwenden könnten, die im Allgemeinen nur von strukturellen Anweisungen in den meisten Grammatiken und Lehrwerken ausgehen.

Andererseits kann gesagt werden, dass die (halb)offene Übung auch, wie erwähnt, als Vorentlastung diente, und auch dazu beitrug, mehr Sicherheit beim Ausfüllen der Lücken zu gewähren. In gleicher Weise kann es sein, dass nicht nur 
die vorherige Aktivität, sondern auch die Verwendung der gleichen Bilder und Beispiele die Motivation der Korrektheit der Lücken verstärkt hat.

\section{Fazit}

Ziel dieser Arbeit war es zu zeigen, dass es möglich ist, Grammatikübungen interessanter und motivierender zu gestalten, insbesondere für Lernende, die ein vertieftes Grammatikwissen benötigen, wie beispielsweise die Lehramtsstudierende einer Deutschausbildung.

Aus einer Öffnungsidee der formfokussierten Übung, das heißt, dass diese Übungen offener waren und zur Reflexion führen können, ist es ersichtlich, dass diese Art Übung für einen Moment der Vorentlastung durchaus interessant ist, weil sie die Reflexion der grammatikalischen Strukturen bereitstellt und den Austausch zwischen Lernenden über das Prinzip der Handlungsorientierung fördert. In ähnlicher Weise zeigte die Umsetzung der Übungen, dass halboffene Aufgaben, über die die Lernenden nachdenken sollen und durch sie ermutigt werden, diese gleichzeitig aber abgelenkt, weswegen sie weniger fokussiert lernen.

Dieses Projekt hat auch bestätigt, was in der Fachliteratur gezeigt wurde, dass ein motivierender und interessanter Unterricht aus mehreren Strategien gewonnen wird, insbesondere durch abwechslungsreiche Übungenstypen. 


\section{Literaturverzeichnis}

BOHL, Thorsten; KUCHARZ, Diemut. Offener Unterricht heute. Konzeptionelle und didaktische Weiterentwicklung. Weinheim: Beltz, 2010.

DÖRNYEI, Zoltán. Engaging language lerners. 19 abr. 2018. (38:37) Verfügbar unter: https://www.youtube.com/watch?v=hg6BSbpVgVU. Zuletzt abgerufen: 18 Mai 2020.

FUNK, Hermann; KUHN, Christina et.al. Aufgaben, Übungen, Interaktion München: Klett-Langenscheidt, 2012.

INSTITUTO GOETHE. Deutsch für den Unterricht. Verfügbar unter: lernplattform.goethe.de>content $>$ VorteileundNachteile. Zuletzt abgerufen: 10 Dezember 2018.

KLEINKNECHT, Marc. Aufgabenkultur im Unterricht. Eine empirischdidaktische Video- und Interviewstudie an Hauptschulen. Baltmannsweiler: Schneider, 2010.

KOUSALOVÁ, Barbora. Motivation und Motivierung im DaF-Unterricht. 2018. Diplomarbeit (Master in Deutsche Sprache und Literatur) - Pädagogische Fakultät, Masaryk Universität, Brünn, 2018.

RIEMER, Claudia. Motivation In: HALLET, Wolfgang; KÖNIGS, Frank G. (Hrsg.): Handbuch Fremdsprachendidaktik. Seelze-Velber: Kallmeyer, 2010, S. 168-172.

ROTH, Gerard. Warum sind Lehren und Lernen so schwierig? Zeitschrift für Pädagogik. Frankfurt am Main, Heft 4, S. 496-506, Juli/August, 2004.

SCHART, Michael; LEGUTKE, Michael. Lehrkompetenz und Unterrichtsgestaltung. München: Klett-Langenscheidt, 2012.

SCHART, Michael. Fach- und sprach-integrierter Unterricht an der Universität: Untersuchungen zum Zusammenspiel von Inhalten, Aufgaben und dialogischen Prozessen. Tübingen: Narr Francke Attempto, 2019.

STARK. R; MANDL, H Konzeptualisierung von Motivation und Motivierung in Konzept situierten Lernens. In: SCHIEFELE, U; WILD, K.P (Hrsg.) Interesse und Lernmotivation; Untersuchungen zu Entwicklung, Förderung und Wirkung. Müster: Waxmann, 2000.

WEINERT, F. E. Leistungsmessungen in Schulen. Weinheim: Beltz.2003. 
WINKLER, U. Differenzierung nach Unterrichtsinhalten am Beispiel von veränderten Aufgabenkulturen: Öffnung von Aufgaben. 201O. Disponível em: www.kopernikus.be.schule.de/downloads/offene aufgaben.pdf. Acesso em: 13 dez 2018.

Artigo recebido em 16 de maio de 2020 e aceito em 23 de junho de 2020.

\section{Anhänge}

\section{BEOBACHTUNGSBOGEN}

\section{Wirkung von (halb)offenen Übungen auf die Motivation der} Lernenden

Klasse: Lingua Alemã II

Phase/Aufgabe: bei den halboffenen Übungen

1. Während der Durchführung der Ưbungen

\subsection{Stimmung:}

( ) Konzentration (x) Interesse (x) Ablenkung ( ) Langeweile ( ) Andere:

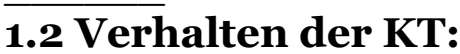

( ) Einzel- bzw. Stillarbeit.

(x) KT fragen sich gegenseitig über die Aufgabe.

(x) KT stellen die Lehrperson Fragen.

( ) KT unterhalten sich über andere Themen.

\subsection{Art von gestellten Fragen:}

(x) nach Wiederholungen.

( ) nach weiteren Aspekten, die über die Ziele der Aufgabe hinausgehen.

(x) nach Aspekten, die nicht direkt um das Thema gehen.

\section{Nach der Aufgabe}




\section{(x) Interesse \\ ( ) Skepis \\ ( ) Andere:}

2.1 Stimmung:

\section{Wirkung von geschlossenen Übungen auf die Motivation der} Lernenden

Klasse: Lingua Alemã II

Phase/Aufgabe: bei den geschlossenen Übungen

\section{Während der Durchführung der Übungen}

\subsection{Stimmung:}

(x) Konzentration (x) Interesse ( ) Ablenkung ( ) Langeweile ( ) Andere:

\subsection{Verhalten der KT:}

(x) Einzel- bzw. Stillarbeit.

( ) KT fragen sich gegenseitig über die Aufgabe.

( ) KT stellen die Lehrperson Fragen.

(x) KT unterhalten sich über andere Themen.

\subsection{Art von gestellten Fragen:}

( ) nach Wiederholungen.

(x) nach weiteren Aspekten, die über die Ziele der Aufgabe hinausgehen.

(x) nach Aspekten, die nicht direkt um das Thema gehen.

\section{Nach der Aufgabe}

\subsection{Stimmung:}

(x) Interesse

( ) Skepis

( ) Andere:

\section{HALBOFFENE ÜBUNG}

\section{DIE ÜBUNGEN}

1) Lesen Sie die Texte: 

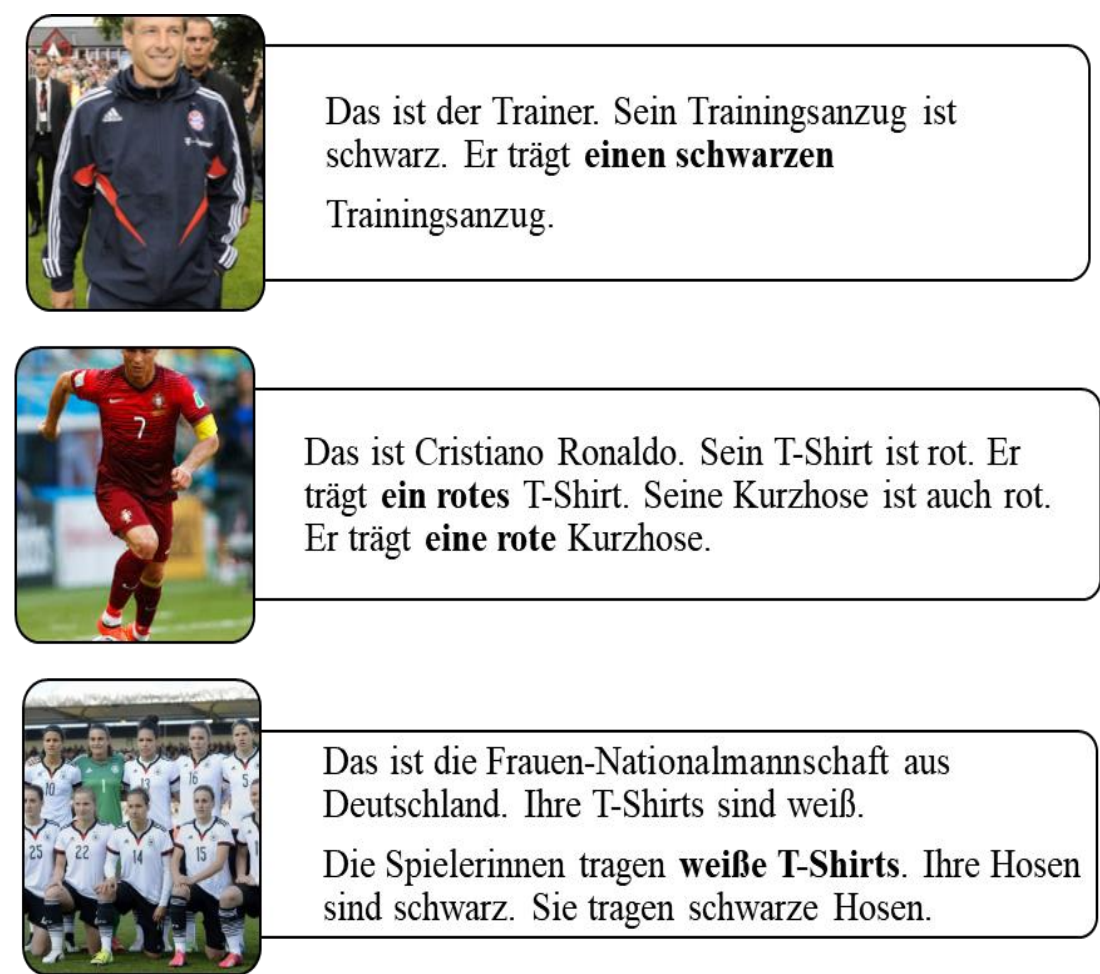

Quelle: FUNK; KUHN. Studio 21. Köln:

2) Vergleichen Sie die Wörter, die fett geschrieben sind. Raten Sie mal, warum sie so aussehen?

3) Erkennen Sie diese grammatikalische Struktur? Was drückt sie aus?

4) Tauschen Sie sich mit Ihrer Nachbarin/Ihrem Nachbarn aus. Was sagen Sie über diese Struktur? Wie erkennt man sie im Satz? Wie wichtig ist sie?

\section{GESCHLOSSENE ÜBUNG}

1) Adjektive im Akkusativ mit unbestimmtem Artikel:

\begin{tabular}{c|ccc}
\multicolumn{2}{c}{ Den } & das & die \\
\hline Singular & $\begin{array}{c}\text { Einen schwarzen } \\
\text { Trainingsanzug }\end{array}$ & $\begin{array}{c}\text { Ein gelbes T- } \\
\text { Shirt }\end{array}$ & Eine blaue Hose
\end{tabular}




\section{Plural Schwarze Anzüge/ T-Shirts/Hosen}

Ergänzen Sie:

einen schwarzen - eine rote - ein rotes - weil̉e - schwarze
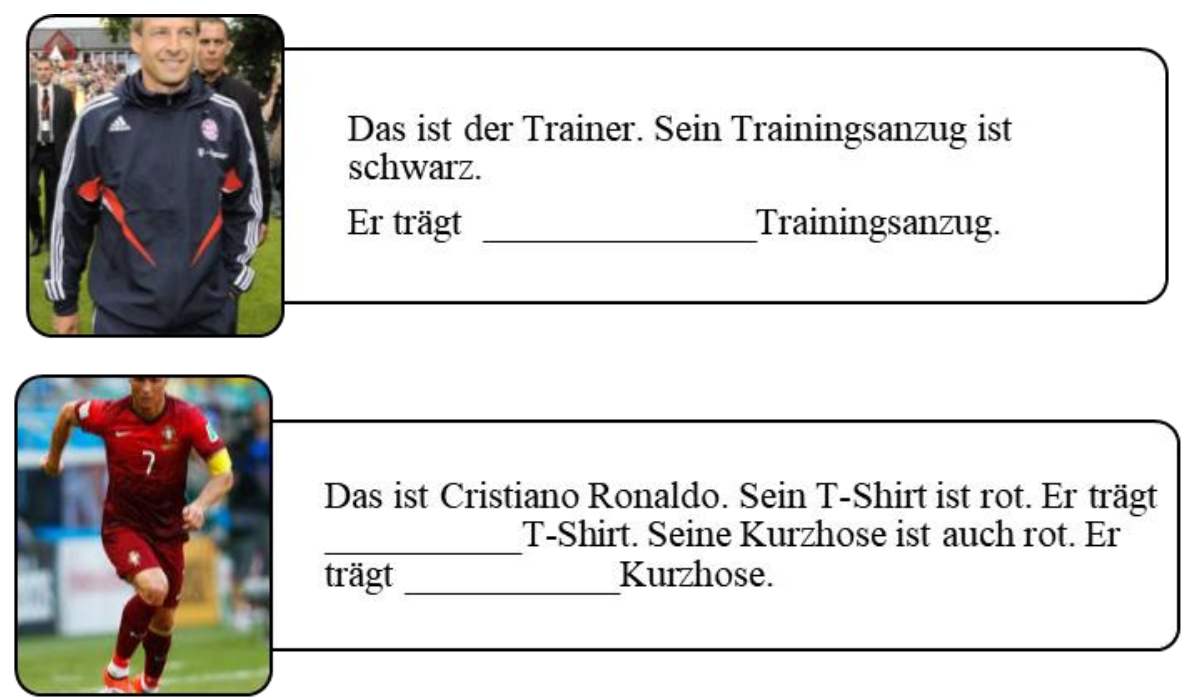

$\begin{aligned} & \text { Das ist die Frauen-Nationalmannschaft aus } \\ & \text { Deutschland. Ihre T-Shirts sind weiß. } \\ & \text { Die Spielerinnen tragen } \\ & \text { Hosen sind schwarz. Sie tragen } \\ & \text { Hosen }\end{aligned}$

